\title{
FRET AND FLIM MICROSCOPY IN THE STUDY OF THE ROLE OF OXIDATIVE STRESS AND APOPTOSIS IN AGING
}

\author{
Yingpei Zhang, Jianhua Zhang, Atsushi Masuda, Marissa Lopez-Cruzan, Victoria Centonze, \\ Ramanujan V. Krishnan and Brian Herman
}

\author{
Department of Cellular \& Structural Biology, University of Texas Health Science Center, San \\ Antonio, Texas 78229-3900, USA
}

Apoptosis is a tightly regulated process that plays a critical role in maintaining tissue homeostasis. Tipping the delicate balance between apoptosis and proliferation towards either may cause disease. Recently, a role for apoptosis in the aging process has been suggested based upon a number of correlative studies that document possible alterations in apoptosis in aged organisms and in numerous disease states that affect the aged (1).

Aging is accompanied by a general decline of physiological function, especially at later stages, and significant increases in the incidence of cancer and other degenerative diseases. It has recently been hypothesized that alterations in apoptosis may contribute to these age-associated changes. However, whether there is a role for apoptosis in the ageing process and how ageing may modify the regulatory machinery of apoptosis remains obscure. It seems very likely that signals that regulate ageing will impact apoptosis and the extent of apoptosis may then impact ageing. However, to date, there has been no direct evidence supporting the existence of such cross-communication between ageing and apoptosis in mammalian system.

While the specific biological causes of aging are still to be determined, it is generally agreed that aging is accompanied by an accumulation of damage produced by mitochondrial oxidative stress to cellular components (2). Aging is associated lowered anti-oxidant defense mechanisms and increased oxidative damage to mitochondria. Removal of oxidatively damaged cells through the orderly process of apoptosis (in contrast to necrosis), should be a high priority for the organism. However, because many of the components of the apoptotic machinery are sensitive to oxidative stress themselves, it is likely that alterations in the rates or apoptosis occur as the organism ages (3). Changes in the rates of apoptosis could have dire consequences for the organism and may underlie many of the age-dependent diseases that occur in society today.

The mitochondrial theory of aging states that oxidative damage to mitochondria over the lifespan of an organism has been hypothesized to contribute to aging. The critical role of the mitochondria in both generating and being a target of ROS and in initiating apoptosis, coupled with the fact that oxidative damage to mitochondrial proteins, lipids and DNA increases with age, suggest that age-dependent oxidative-induced alterations in mitochondrial function and their attendant effects on apoptosis, may play a role in the regulation of the aging process. We have been examining the role of oxidative stress, the mitochondria and apoptosis in aging. We have observed increased activities of two caspases, caspas e-2 and caspase-9, which are involved in mitochondria-mediated apoptosis in livers isolated from old rats, and found that hepatocytes isolated from old animals ( $>23$ months) are more sensitive to oxidative stress that targets the mitochondria compared to those isolated from young (6 month) animals (4).

Caspase-2 and -9 are both considered initiator caspases, activated as part of the early events in apoptosis. Other investigators have suggested that certain caspases may be associated with mitochondria (5). To determine whether mitochondria contain caspase- 2 activity that can be activated by oxidative stress, we transiently transfected cells with mitochondrially targeted constructs containing tandem cyanine and yellow fluorescent proteins linked by caspase substrate or by non-cleavable glycine residues. To examine oxidative stress-induced activation of caspase- 2 using this substrate, we employed the technique of Fluorescence Resonance Energy Transfer (FRET). FRET is a process by which a donor fluorophore in an excited state may transfer its excitation energy to a neighboring acceptor fluorophore nonradiatively through dipole-dipole interactions. For FRET to occur, the emission spectrum of the donor must overlap with the absorption spectrum of the acceptor. Energy transfer varies as the inverse of the sixth power of the distance separating the donor and acceptor and for efficient FRET, the distance between donor and acceptor molecules must be between $10-50 \AA$. 
Methods to detect FRET may monitor changes in donor and acceptor emission or changes in the intrinsic fluorescence lifetime of the donor molecule. We have developed a new technology streak camera detection system based upon the use of a streak camera detector to monitor changes in caspase-2 activation following oxidative stress (Figure 1). FRET is measured as the difference in the lifetime of the donor in the presence and absence of the acceptor. FRET leads to quenching (shortening) of the donor lifetime.

\section{Figure 1. Functional (A) and Optical (B) layout of the Multiphoton StreakFLIM system. G1: Horizontal Galvo Mirror; G2 : Vertical Galvo Mirror ; L1: Focusing relay lens; $C$ : Coupling lens for sideport optics; I : Imaging lens; $O$ : Microscope Objective lens; D1: Dichroic; PC :Photocathode;}

\section{Streak-FLIM System}
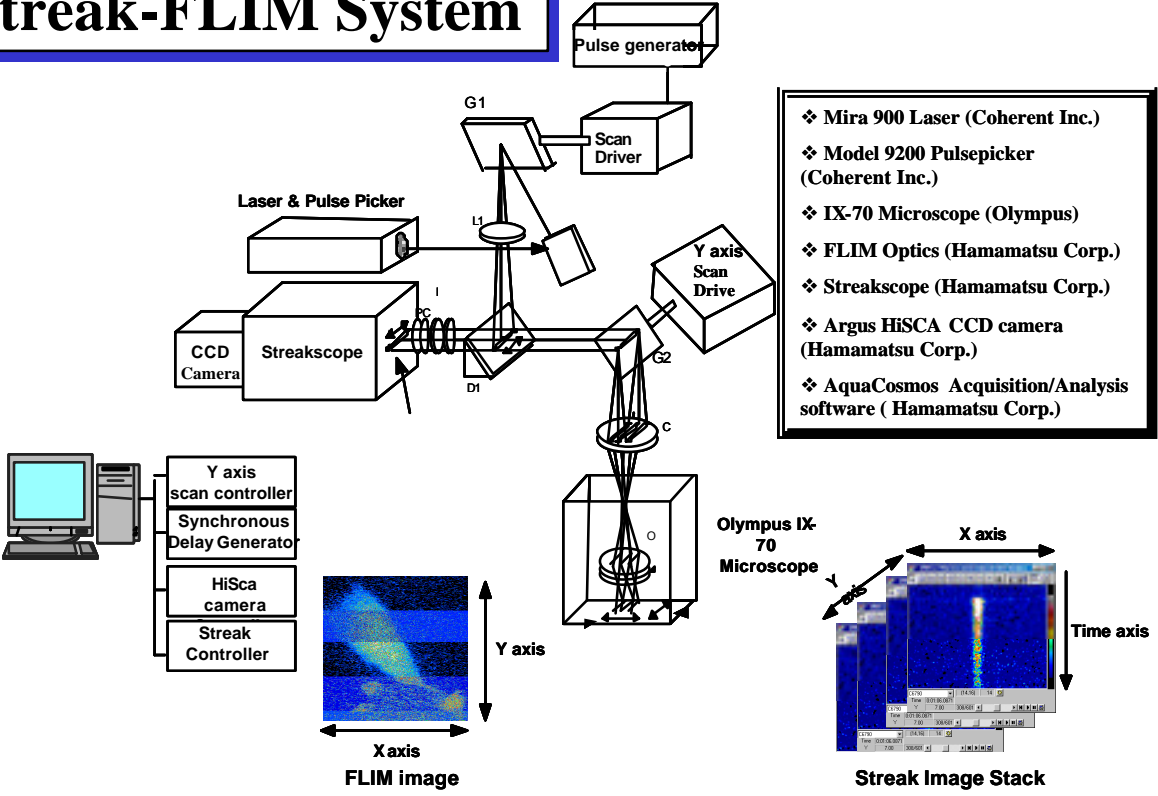

The lifetime on CFP in BHK cells expressing either mitochondrially targeted CFP alone (mCFP), mitochondrially targeted CGY (mCGY) or mitochondrially targeted C2Y (mC2Y) in the presence or absence of t-butylhydroperoxide ( $\mathrm{t}$ $\mathrm{BOOH}$ ) was measured using the streakFLIM system (Figure 2). mCFP yields the intrinsic lifetime of CFP in the absence of any FRET. mCGY yields the lifetime of CFP under conditions of maximal FRET in the absence of cleavage of caspase- 2 as this construct contains a non-cleavable linker between the CFP and YFP proteins. $\mathrm{mC} 2 \mathrm{Y}$ yields the lifetime of the caspase- 2 under normal and oxidative stress $(+\mathrm{t}-\mathrm{BOOH})$. As can be seen, $\mathrm{t}-\mathrm{BOOH}$ treatment results in an increase in the lifetime of $\mathrm{mC} 2 \mathrm{Y}$ signifying activation of caspase- 2 and subsequent cleavage of the caspase-2 substrate.
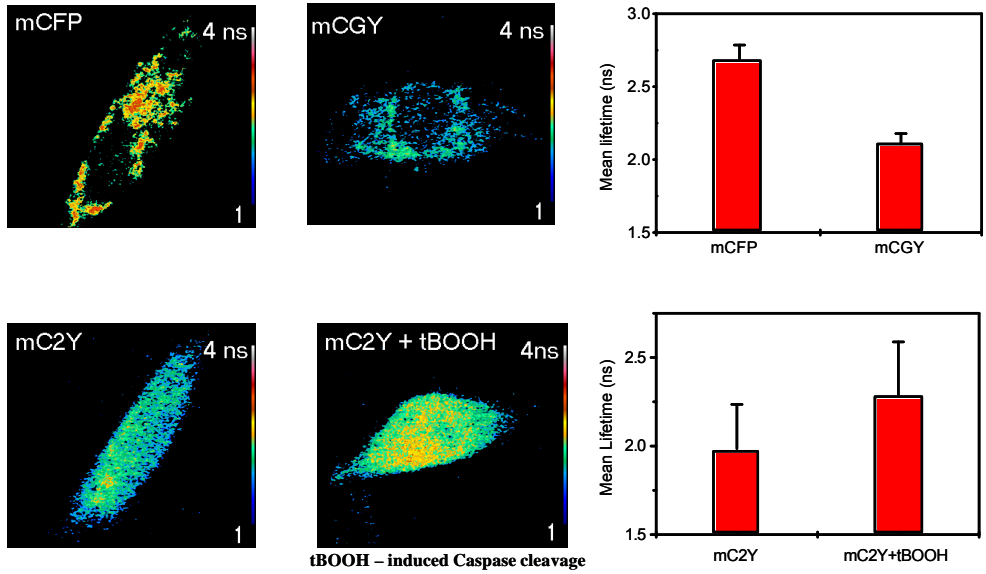

Figure 2. Lifetime of CFP in living cells. The lifetime of CFP was determined in cells expressing mitochondrially targeted CFP, non-cleavable CGY and C2Y in the presence and absence of t-BOOH exposure $\left(50 \mu \mathrm{M}, 4 \mathrm{hr}, 37^{\circ} \mathrm{C}\right)$.

References:

1) Warner, H.R., Ann N Y Acad Sci 88, (1999) 1.

2) Honda, Y., Honda, S., 1999. FASEB J. 13, (1999) 1385.

3)Hampton, M.B., Orrenius, S., FEBS Lett. 414, (1997) 552.

4) Zhang, Y., Chong, E., and Herman, B. Exptl. Gerontol. 37: (2002) 777.

5) Krajewski, S., Krajewska, M., Ellerby, L.M., welsh, K., Xie, Z., Deveraux, O.L., Slavesen, G.S., Bredesen D.E., Rosenthal, R.E., Fiskum, G., and Reed, J.C. Proc. Natl. Acad. Sci. U.S.A. 96: (1999) 5752. 